\title{
On the Use of Fuzzy Rule Interpolation Techniques for Monotonic Multi-Input Fuzzy Rule Base Models
}

\author{
Kai Meng Tay ${ }^{1 *}$, Chee Peng Lim ${ }^{2}$
}

\begin{abstract}
Constructing a monotonicity relating function is important, as many engineering problems revolve around a monotonicity relationship between input(s) and output(s). In this paper, we investigate the use of fuzzy rule interpolation techniques for monotonicity relating fuzzy inference system (FIS). A mathematical derivation on the conditions of an FIS to be monotone is provided. From the derivation, two conditions are necessary. The derivation suggests that the mapped consequence fuzzy set of an FIS to be of a monotonicity order. We further evaluate the use of fuzzy rule interpolation techniques in predicting a consequent associated with an observation according to the monotonicity order. There are several findings in this article. We point out the importance of an ordering criterion in rule selection for a multi-input FIS before the interpolation process; and hence, the practice of choosing the nearest rules may not be true in this case. To fulfill the monotonicity order, we argue with an example that conventional fuzzy rule interpolation techniques that predict each consequence separately is not suitable in this case. We further suggest another class of interpolation techniques that predicts the consequence of a set of observations simultaneously, instead of separately. This can be accomplished with the use of a search algorithm, such as the brute force, genetic algorithm or etc.
\end{abstract}

\section{INTRODUCTION}

Many real world problems involve systems that satisfy the monotonicity property between input(s) and output(s). In this paper, we assume that specific monotonicity preserving modeling techniques can give a better approximation than that of general modeling techniques, particularly for monotonicity relating problems. For example, various Hermite interpolation techniques are introduced as specific interpolation techniques for curve fitting problems that involve the monotone property. A fuzzy rule base model, or fuzzy inference system (FIS) has been proven to be a universal approximator that can approximate any function to any degree of accuracy [1]. From the literature review, various investigations have been conducted to study the monotonicity property in FISs. For example, Zhao analyzes on the conditions of a Mamdani fuzzy Model to be monotone [2]. We have also shown the importance of the monotonicity property in FISbased assessment models in our previous work [3, 4].

It has been a new trend to use fuzzy rule interpolation techniques as a solution to incomplete rule base in fuzzy modeling [5, 6]. Getting a complete rule base is not possible all the time, especially for multi-input FISs, as the number of rules required is huge. One of the most important assumptions in fuzzy rule interpolation techniques is to assume all state variables are bounded and

Kai Meng Tay is now with the University Malaysia Sarawak, Malaysia. (Corresponding author, email: kmtay@feng.unimas.my ).

Chee Peng Lim is now with the University of Science Malaysia, Malaysia. (email: cplim@eng.usm.my). gradual, ordering in all variables exists, and the concept of fuzzy distance is applicable.

Over the years, various improvements of fuzzy rule interpolation techniques have been proposed. The improvements include compatibility with rule base, avoiding abnormal fuzzy set, convexity, normalization, linearity preservation, and variety in membership functions at the input or output space. However, to the best of our knowledge, no investigation on the use of fuzzy rule interpolation techniques in monotonicity preserving FIS has been reported. This further motivates us to examine the use of fuzzy rules techniques in monotonicity relating function approximation problems.

Our investigation starts with a derivation of the conditions for a multi-input FIS to fulfill the monotonicity property with differentiation of its output with respect to its input(s). From the derivation, a set of sufficient conditions is obtained. At the antecedent part, a method to tune the membership functions is suggested. At the consequence part, the derivation stresses on the importance of having a monotone rule base for modeling of a monotone function. In this paper, these conditions are used to develop a monotonic FIS.

From the derivation, firstly, we analyze the ability of fuzzy rule interpolation techniques that is based on the fundamental equation of rule interpolation (FERI) to produce a consequence association which is comparable with that of existing fuzzy rules. This analysis is meaningful, as most of the fuzzy rule interpolation techniques are developed based on FERI. Our analysis points out the importance of having an ordering criterion in the rule selection process. We further propose an ordering criterion for multi-input fuzzy rule base models.

Secondly, we analyze the ability of a fuzzy rule interpolation technique to produce predicted consequences which are comparable among each other. We further point out that fuzzy rule interpolation that predicts each consequence separately with piecewise interpolation techniques may not be a solution for monotonicity relating multi-input fuzzy rule base models. We then re-formulate this problem, and argue the importance of another class of fuzzy rule interpolation technique that predicts the consequence of a set of observations simultaneously, instead of separately.

The organization of this paper is as follows. Section II explains the representative value of fuzzy sets, ordering and distance of fuzzy sets, and a multi-input FIS. Section III discusses a generalization of fuzzy rule interpolation techniques. Section IV presents the derivation of a sufficient conditions of a monotone FIS. In Section V, applicability of fuzzy rule interpolative techniques in FIS models is investigated. Finally, concluding remarks are presented in Section VI. 